\title{
In-situ measurement of annealing kinetics of individual bulk grains in nanostructured aluminium
}

Wu, G.L.; Juul Jensen, Dorte

Published in:

Philosophical Magazine

Link to article, DOI:

$10.1080 / 14786435.2012 .711494$

Publication date:

2012

Document Version

Early version, also known as pre-print

Link back to DTU Orbit

Citation (APA):

Wu, G. L., \& Juul Jensen, D. (2012). In-situ measurement of annealing kinetics of individual bulk grains in nanostructured aluminium. Philosophical Magazine, 92(25-27), 3381-3391.

https://doi.org/10.1080/14786435.2012.711494

\section{General rights}

Copyright and moral rights for the publications made accessible in the public portal are retained by the authors and/or other copyright owners and it is a condition of accessing publications that users recognise and abide by the legal requirements associated with these rights.

- Users may download and print one copy of any publication from the public portal for the purpose of private study or research.

- You may not further distribute the material or use it for any profit-making activity or commercial gain

- You may freely distribute the URL identifying the publication in the public portal

If you believe that this document breaches copyright please contact us providing details, and we will remove access to the work immediately and investigate your claim. 
Title: In-situ measurement of annealing kinetics of individual bulk grains in nanostructured aluminium

Authors: Guilin Wu* and Dorte Juul Jensen

(* Corresponding author)

\section{Affiliations:}

Guilin Wu: College of Materials Science and Engineering, Chongqing University, 400045, Chongqing, China; Tel: (+86) 236511 1295; Fax: (+86) 236511 1295; Email: guilin.wu@gmail.com.

Dorte Juul Jensen: Danish-Chinese Center for Nanometal, Materials Science and Advanced Characterization Section, Department of Wind Energy, Risø Campus, Technical University of Denmark, DK-4000, Roskilde, Denmark; Tel: (+45) 4677 5701; Fax: (+45) 4677 5758; Email: doje@dtu.dk. 


\title{
In-situ measurement of annealing kinetics of individual bulk grains in nanostructured aluminium
}

\author{
G. L. $\mathrm{Wu}^{1}$ and D. Juul Jensen ${ }^{2}$
}

1 College of Materials Science and Engineering, Chongqing University, 400045, Chongqing, China

2 Danish-Chinese Center for Nanometal, Materials Science and Advanced Characterization Section, Department of Wind Energy, Risø Campus, Technical University of Denmark, DK-4000, Roskilde, Denmark

\begin{abstract}
Non-destructive 3 dimensional X-ray diffraction (3DXRD) is used to characterize the coarsening and growth of bulk crystallites in very heavily deformed aluminium in-situ during isothermal annealing. It is found that initially during the annealing, coarsening by recovery dominates. Later recrystallisation starts and by fast growth of recrystallisation nuclei, some very big grains evolve. This occurs simultaneously with recovery coarsening other parts of the microstructure. Consequently very broad grain size distributions are observed. The 3DXRD results shows that the size of the nuclei (those crystallites that end up being very large by fast recrystallisation growth) do not have an initial size advantage compared to those coarsening much slower by recovery. Kinetics curves for these 2 categories of grains are determined. Data of this type are considered very important for understanding the thermal response of nanometals and thus also for instructing thermal treatment for optimal mechanical properties.
\end{abstract}


Keywords: Nanometals; Aluminium; Annealing; Kinetics; 3DXRD.

\section{Introduction}

Nanostructured metals and alloys prepared by plastic deformation to very high strains have attracted keen interest recently. A general pattern for the microstructural evolution during plastic deformation has been established [1-2]. Lamellar dislocation boundaries are observed at high strains, the misorientations across which are typically high $\left(>15^{\circ}\right)$. With increasing strain the fraction of high angle boundaries increases to be as high as 60$80 \%$ [3-4] and the distance between these boundaries are as small as $300 \mathrm{~nm}$ in $\mathrm{Al}$ [5-6] and may be even smaller in other materials, e.g. Ni [7].

Nanostructured metals and alloys offer substantially increased strength but usually with a much reduced ductility. In single phase materials, annealing treatment bas been suggested as a way to improve the ductility without too much loss of strength [8]. It has however also been found that annealing after deformation to very high strain can lead to a strengthing of the materials and even less ductility than in the deformed state [9]. Furthermore the nanostructured metals and alloys may be thermally unstable even at room temperature [10]. Therefore, in order to exploit these materials in practical terms, thermal treatment need to be investigated, and the underlying mechanisms understood, such that a complete basket of properties may be optimized. A problem when addressing this question is that with standard experimental methods it is only possible to follow the 
annealing kinetics for sample surfaces [11] which may not be typical for the bulk, or to do ex-situ statistical analysis of different samples annealed for different times [12].

In the present study we have used a unique technique - 3 dimensional X-ray diffraction (3DXRD) microscopy, which allows in-situ, non-destructive measurements of the volume increase of individual bulk crystallites to quantify the kinetics for many individual growing crystallites in the bulk of aluminium cold-rolled to a strain of 4.0 (i.e. reduced in thickness by 98.2 \%). Details on the 3DXRD microscopy are given in [13-14]. This method has not previously been used for studies of the annealing response of nanometals but only for metals deformed to lower strains, i.e. for coarser-scale structures [15-16]. We show that in $\mathrm{Al}$ cold rolled to a strain of 4.0 coarsening by recovery occurs simultaneously with recrystallisation and it is suggested that the coarsening is necessary to initiate recrystallisation in this material.

\section{Experimental}

The starting material is a commercial purity Al alloy AA1200 with a chemical composition of $99.1 \% \mathrm{Al}-0.59 \% \mathrm{Fe}-0.13 \% \mathrm{Si}-0.12 \% \mathrm{Cu}$ (weight percent), which was provided by Alcan. The material was cold-rolled to a strain of 4 (98.2 \% reduction in thickness) by industrial standards. The reason for choosing this material is that the deformation microstructure has been extensively studied by transmission electron microscope [6] and high resolution electron backscatter diffraction (EBSD) techniques. The deformation microstructure is given in Fig. 1. It is seen (Fig. 1a) that the deformed microstructure is mainly composed of extended lamellar boundaries approximately 
parallel to $\mathrm{RD}$ ( $\mathrm{RD}$ being the rolling direction during the rolling process) with short interconnecting boundaries. The spacings of lamellar boundaries vary from $100 \mathrm{~nm}$ to $1-2$ $\mu \mathrm{m}$, with the average of about $340 \mathrm{~nm}$. These features are typical of the microstructure of aluminium deformed to high strains [6].

3DXRD was used to determine the annealing kinetics of individual crystallites in the bulk in-situ while annealing the sample. The 3DXRD method was developed with the purpose of non-destructive bulk kinetics investigation. This requires high penetration power as well as fast measurements. High energy (50-100 keV) X-rays give the necessary penetration power ( $\mathrm{mm}-\mathrm{cm}$ in typical materials) and several experimental approaches have been developed for full 3D mapping of the microstructure [17-20]. However these methods rely on scanning principles to obtain the full 3D information, which typically makes them too slow for in-situ kinetics investigation. 3DXRD is a different method, which uses tomography principles to get the $3 \mathrm{D}$ information and only requires sample rotation, the measurement are thus much faster. For further details see [13].

The experiment was performed using the beamline ID11 at the European Synchrotron Radiation Facility (ESRF) at Grenoble, France. The experimental set-up of the present insitu annealing experiment is the same as that described in [15]. For clarity, the set-up is sketched in figure 2. An as-deformed specimen with sizes of $6 \mathrm{~mm}$ along RD, $4 \mathrm{~mm}$ along the transverse direction (TD) and $500 \mu \mathrm{m}$ along the normal direction (ND) is mounted with the ND parallel to the beam and RD upwards in a small X-ray transparent furnace mounted on a $\omega$ rotation stage. (Here $\omega$ is the rotation angle around the vertical 
axis, see Fig. 2.) The sample is isothermally annealed at $257^{\circ} \mathrm{C}$ for in total $20 \mathrm{~h}$ in an argon atmosphere. A focused monochromatic X-ray beam with an energy of $54 \mathrm{keV}$ and dimensions of $100 \times 100 \mu \mathrm{m}^{2}$ is used to illuminate the sample during the annealing. The sample gauge volume thus is $100 \times 100 \times 500 \mu \mathrm{m}^{3}$. Grains growing out of this volume are not included in the analysis. This means that the kinetics of the few grains which grow to become very large (see Fig. 1c) as well as crystallites near the edge of the gauge volume are not followed here. Diffraction images are taken by a 2048×2048 pixels CCD detector repeatedly at 300 consecutive $\omega$ settings with a step size of $0.1^{\circ}$ and an oscillation angle of $\Delta \omega=0.1^{\circ}$ at each scanning step. The $\omega$-range spans $30^{\circ}$ to cover a large orientation space. Two scans, with $1 \mathrm{~s}$ and $3 \mathrm{~s}$ exposure times, respectively, are taken during the in-situ annealing experiment. This was to ensure that both large and small grains could be well recorded. However the data analysis later revealed that the scans with $1 \mathrm{~s}$ exposure time gave good results for crystallites as small as $1 \mu \mathrm{m}$. So only these scans are used for the present results. The time resolution for the scan with $1 \mathrm{~s}$ exposure time, including exposure, recording images and rotating the stage, is about 15 min, and the time resolution for the other scan is longer. Therefore a temporal resolution of $0.7 \mathrm{~h}$ between successive scans is obtained. Given by the present experimental setup and the method for analysing the data, the smallest crystallite size that can be determined is equal to $\sim 1 \mu \mathrm{m}$. For further details on the experimental procedure, see [15].

Crystallites fulfilling Bragg's law at a given $\omega$ angle within the illuminated gauge volume $\left(100 \times 100 \times 500 \mathrm{~m}^{3}\right)$ were seen in the detector images as diffraction spots. Initially in the deformed stage, the nanostructured matrix leads to many spots originating from the 
deformed matrix. As annealing progressed, the intensities of many spots increase. The integrated intensities of spots are obtained by summing the pixel intensities and subtracting the background. It is adequate to start analysing the diffraction pattern after $20 \mathrm{~h}$ annealing when spots in this series of patterns (300 $\omega$ settings) are clearly identified. These spots are then traced back through all diffraction patterns corresponding to different annealing times and the size evolutions (i.e. coarsening kinetics) for the corresponding crystallites are obtained. This procedure is relative simple and robust and can avoid a too serious problem of spot overlap which is severe in the early stage. This means we can relatively well differentiate spots, i.e. crystallites in the initial deformed sample. It is important to note that with this analysis procedure only the crystallites surviving the annealing are characterized, the ones that shrink and disappear - leaving room for the growing ones - are not included. However, spot overlap is still a problem for the early scans. To get reasonable reliable data at these stages an algorithm of multiple Gauss curve fitting was applied (see the appendix for more details). Since the integrated intensities of spots are proportional to the volume of the associated crystallites, the growth kinetics of individual crystallites can be determined by measuring the integrated spot intensities as a function of annealing time. The intensities may be converted to volumes by the method described in [21]. However, here a simpler method is used: since the present material has been characterized in detail by electron back scatter diffraction (EBSD) using a HKL Channel 5 detector attached to a Zeiss Super 35 thermal field emission gun scanning electron microscope - the average sizes of crystallites at given times are used to calibrate the relationship between X-ray intensity and crystallite volume. 


\section{Results and Discussions}

In total, 170 valid spots corresponding to 170 growing crystallites are found and analyzed. It was found that for many crystallites only a rather subtle growth or coarsening is observed leading to sizes in the range of $3 \mu \mathrm{m}$ to $7 \mu \mathrm{m}$. Some crystallites, however, grow to become large ( $\geq 14 \mu \mathrm{m})$ and the remaining crystallites grow to intermediate sizes. Figure 3a gives an example of the recrystallisation kinetics of 10 crystallites with different finial sizes. The figure further shows that each crystallite has its own kinetics. This is also observed for recrystallisation of metals deformed to lower strains [22-23] and has been ascribed to the fact that the recrystallising crystallites grow in a heterogeneous deformation microstructure, where the surroundings of the crystallites change in space and time while the crystallite is growing. The present deformation microstructure is heavily subdivided and fairly inhomogeneous (see Fig. 1a) and it is therefore not surprising that each crystallite has its own kinetics as each crystallite will meet different surroundings; but it also underlines the importance of including distributions of growth rates rather than just one growth rate for all crystallites in modelling of metals deformed to very high strains [24].

It should be pointed out that many of crystallites are too small initially to give data in the first scan (annealing time $0 \mathrm{~h}$ ), so many of the crystallites are only detected in the second (annealing time $0.7 \mathrm{~h}$ ) and subsequent scans, see for example grain No. 2 and No. 8 in Fig. 3a. It was found that only 60 crystallites are of a sufficient size to be detected in the first and all subsequent scans. By comparing the kinetics of all 170 crystallites and those 
of the 60 crystallites, it is however found that the behaviour of the 2 groups is very similar.

After $20 \mathrm{~h}$ annealing, a broad size distribution is observed (see Fig. 3b). For further analysis, the 170 crystallites are subdivided into two categories: large crystallites with final sizes larger than the average value $(9.8 \mu \mathrm{m})$ and small crystallites with final sizes smaller than the average. These categories are used in Fig. 4 where the distribution of sizes after annealing for $0.7 \mathrm{~h}$ is shown. (The crystal sizes after $0.7 \mathrm{~h}$ annealing were used because many crystallites are too small to be detected at the very beginning of annealing as described above. In the following $0.7 \mathrm{~h}$ is referred as the early stage.) It is seen that the early crystallite sizes varies over a wide range. Furthermore it can be seen that those crystallites that end up being large (above the average size) are not all large in the early stage of annealing.

If for simplicity we define all crystallites below the average size after $20 \mathrm{~h}$ annealing as recovering and the ones above as recrystallising, we can determine the growth rates of these two classes during the entire annealing process. It has to be noted that this classification is not fully correct as some recrystallising grains may end up being small due to impingement with other recrystallising grains, but it serves to illustrate important differences between the 2 classes. During early stages of annealing $(0-2.1 \mathrm{~h})$, all crystallites are coarsening. The growth/coarsening kinetics is similar for the two groups. Figure $5 \mathrm{a}$ shows the plot of growth rates versus sizes of recovering and recrystallising crystallites after $0.7 \mathrm{~h}$ annealing. It is seen that the sizes and growth rates of all 
crystallites vary over a wide range. The annealing behaviour of all crystallites at this stage can be described to our best knowledge as recovery. Figure 5b shows the plot of sizes versus growth rates of all recovering and recrystallising crystallites after $5.0 \mathrm{~h}$ annealing. It is seen that recovering crystallites concentrate in the range with small sizes and low growth rates. Recrystallising crystallites however are distributed in the ranges having either large sizes or fast growth rates. The average growth rates of recovering crystallites and recrystallising crystallites during the whole annealing process is plotted in figure 5c. It is clear that the growth/coarsening rates of recovering crystallites decrease constantly during annealing. The growth rates of recrystallising crystallites on the other hand increase up to $2.1 \mathrm{~h}$ annealing after which the growth rates are reduced. The latter is likely to be due to impingement effects.

The explanation for the wide size distribution observed after annealing for $20 \mathrm{~h}$ (Fig. 3b) may relate to concurrent recovery and recrystallisation which have been reported in a similar sample [25-26]. In that work, it was found that initially recovery but later recrystallisation dominated. This may also be seen in the present data that in the first 0.7 $\mathrm{h}$ annealing all crystallites coarsen at a not too different rate, but after that - essentially in the time period from $0.7 \mathrm{~h}$ to about $2.1 \mathrm{~h}$ a few of the crystallites are observed to grow much more rapidly than the others ending up becoming very large. The rest of the crystallites just continue to coarsen at low rates.

The in-situ 3DXRD results match the microstructure observed destructively in a same set samples annealed for different times (see Fig. 1b). After $5.0 \mathrm{~h}$ anneal the microstructure 
consists of relatively few large crystallites free of interior dislocation boundaries, many small crystallites also free of interior boundaries and deformed matrix. The deformed material in figure $1 \mathrm{a}$ and figure $1 \mathrm{~b}$ is seen as areas filled with white and black lines whereas the other more strain free crystallites are seen as areas with just one colour (i.e. each with one crystallographic orientation) without interior subdivisions and partly or fully surrounded by a high angle boundary ( $>15^{\circ}$ misorientation). The 3DXRD experiment was stopped after $20 \mathrm{~h}$ and the microstructure in this stage is shown in figure 1c. It is almost free of deformed materials and consists of a wide distribution of grain sizes from very small $\sim 3 \mu \mathrm{m}$ to very large $\sim 100 \mu \mathrm{m}$.

Combining the information from the 3DXRD and the microscopy investigation thus show that both recovery and recrystallisation occur in this material. The annealing process starts with rapid recovery leading to a coarsening of the structure. After some coarsening has occurred, a few nuclei have developed which then start to grow quickly in a typical recrystallisation process, while in other parts of the structure many crystallites just continue to coarsen by recovery. In this latter stage, however, recrystallisation dominates as the recrystallising crystallites accounts for by far the largest fraction of the sample volume.

What the present 3DXRD experiment reveals which is new compared to previous investigation is that recrystallisation only takes place after an initial quite extended period of recovery at elevated temperature. Typically in $\mathrm{Al}$ of the same purity deformed to lower strain $\varepsilon=2.0$ and 2.3 [27-28], site saturation was observed, i.e. all nuclei form 
immediately upon annealing (or at least at a very early stage). In heavily deformed $\mathrm{Al}$ ( $\varepsilon$ $=4.0$ ) an analysis of hardness data has shown that recovery has activation energies in the range $89-125 \mathrm{~kJ} / \mathrm{mol}$ whereas recrystallisation has an activation energy equal to 178 $\mathrm{kJ} / \mathrm{mol}[25]$.

During the initial recovery the structure coarsens and becomes more heterogeneous [5], whereby recrystallisation may be encouraged. This together with the fact revealed by the present 3DXRD result, that rapid recrystallisation growth only occurs after a period of significant recovery suggests that the recovery is necessary before nuclei can form and grow rapidly, i.e. before recrystallisation can start in the present material.

\section{Conclusions}

Three dimensional X-ray diffraction has been used to follow the kinetics of individual bulk crystallites in-situ during annealing of aluminium deformed by cold rolling to a strain of 4. It is shown that the annealing leads to simultaneous recovery and recrystallisation, and that initially crystallites which later become recrystallising grains, are not always bigger than those that just continuously coarsen by recovery. Recrystallisation only starts after an extended period of recovery and recovery continues in the non-recrystallised parts of the structure during the entire annealing period. It is argued that the initial recovery is needed for the formation of nuclei above the critical size. Within the 2 groups, recovery and recrystallisation crystallites, large variations in kinetics are observed. The complex interplay between recovery and recrystallisation as observed in the present sample may be used for optimising the properties (e.g. strength 
and ductility) of materials similar to the present which are generally referred to as nanometals.

\section{Acknowledgement}

The authors wish to thank Drs. L. Margulies, E.M. Lauridsen, S.S. West and R.B. Godiksen for their assistance in the experiment and Drs. B. Ralph, N. Hansen, X. Huang, A.W. Godfrey and Y.B. Zhang for their comments and discussions. The authors gratefully acknowledge financial support from the National Natural Science Foundation of China (NSFC) (Grant No. 51001016) and Danish National Research Foundation and the NSFC (Grant No. 50911130230) for the Danish-Chinese Center for Nanometals. GW would also like to acknowledge the finical support from the Young Merit Scholar of Chongqing University. ESRF is greatly acknowledged for the usage of synchrotron facilities. 


\section{Appendix}

During in-situ annealing, the diffraction images were obtained by a 2 dimensional Charged Couple Device (CCD) detector with a resolution of 2048 pixels by 2048 pixels. An example of an image is shown in figure A1 (a). Crystallites fulfilling Bragg's law at a given $\omega$ rotation within the illuminated gauge volume are seen in the images as diffraction spots against a background of much more diffuse Debye-Scherer rings originating from the deformed matrix with a strong rolling texture. As annealing proceeds, spots appear and their intensities increase as crystallites develop and grow during annealing. The intensities of diffraction spots is determined by plotting the intensities of all pixels along the Debye-Scherrer rings. Thus each Debye-Scherrer ring is converted into a line profile (or intensity spectrum) and the background is subtracted. The volumes of crystallites are obtained by integrating the intensities of peaks. A major problem is however spot overlap, which is typical for textured sample. An automatic algorithm has to be developed to overcome this problem.

It is assumed that the intensity of each peak in the spectrum can be described by a Gauss function. Therefore a multiple Gauss curve fitting procedure is compiled to separate the overlapping peaks and get the integrated intensity of each peak. In a Gauss curve fitting, normally there are three parameters to be fitted, i.e. the amplitude, the centroid (location) and the standard error related to the peak width. An example is given in figure A1 (b) which shows the pixel intensity after background subtraction along the Debye-Scherrer ring. In the figure, 6 peaks are seen overlapping. The algorithm for the given example 
runs as follow: 1) identify each peak (local maximum) and estimate its width and maximum position in the spectrum; 2) make the first guess of the 3 parameters and run a single Gauss curve fitting for each peak; 3) calculate the intensity of the 6 peaks and adjust the parameters using the intensity differences between the experimental data and fitted peaks; 4) use the adjusted parameter instead of the guess in step 2 and iterate steps 2 and 3 until the smallest fitting error is obtained. By applying the multiple Gauss curve fitting procedure, the 6 peaks are successfully separated (indicated by the colour lines) and their integrated intensities are obtained.

It should be pointed out that beside Gauss function, other functions, e.g. Lorentz function, can also be defined to describe the intensity of each peak, and there is no limitation in the number of peaks to be fitted in the algorithm. 


\section{References}

[1] N. Hansen, Metall. Trans. A 32 (2001) p.2917.

[2] Y. Saito, H. Utsunomiya, N. Tsuji, T. Sakai, Acta Mater. 47 (1999) p.579.

[3] O.V. Mishin, D. Juul Jensen, N. Hansen, Mater. Sci. Eng. A 342 (2003) p.320.

[4] H. Jazaeri, F.J. Humphreys, Acta Mater. 52 (2004) p.3251.

[5] Q.F. Xing, X. Huang, N. Hansen, Metall. Mater. Trans. A 37 (2006) p.1311.

[6] Q. Liu, X. Huang, D. J. Lloyd, N. Hansen, Acta Mater. 50 (2002) p.3789.

[7] H.W. Zhang, X. Huang, N. Hansen, Acta Mater. 56 (2008) p.5451.

[8] E. Ma, Scripta Mater. 49 (2003) p.663.

[9] X. Huang, N. Hansen, N. Tsuji, Science 312 (2006) p. 249.

[10] O.V. Mishin, V.Y. Gertsman, R.Z. Valiev, G. Gottstein, Scripta Mater. 35 (1996) p.873.

[11] D. Juul Jensen, M.D. Lund, A.W. Larsen, J.R. Bowen, Mater. Sci. Forum 467-470 (2004) p.147

[12] G. Wu, D. Juul Jensen, Mater. Sci. Tech. 21 (2005) p.1407.

[13] H.F. Poulsen, Three-Dimensional X-Ray Diffraction Microscopy: Mapping Polycrystals and their Dynamic, Springer, Berlin, 2004.

[14] D. Juul Jensen, H.F. Poulsen, Å. Kvik, Encyclopedia of Materials: Science and Technology Updates, Elsevier, Oxford, 2005.

[15] E.M. Lauridsen, H.F. Poulsen, S.F. Nielsen, D. Juul Jensen, Acta Mater. 51 (2003) p.4423.

[16] S. Schmidt S, S.F. Nielsen, C. Gundlach, L. Margulies, X. Huang, D. Juul Jensen, Science 305 (2004) p.229. 
[17] B.C. Larson, W.G. Yang, G.E. Ice, J.D. Budai, J.Z. Tischler, Nature 414 (2002) p. 887.

[18] P. Bleut, E. Welcomme, E. Dooryhee, J. Susini, J. -L. Hodeau, P. Walter, Nature Mater. 7 (2008), p.468.

[19] H.J. Bunge, H. Klein, L. Wcislak, U. Garbe, W. Weiss, J.R. Schneider, Textures and Microstr. 35 (2003) p.253.

[20] T. Wroblewski, O. Clauss, H.-A. Crostack, A. Ertel, F. Fandrich, Ch. Genzel, K. Hradil, W. Terne, E. Woldt, Nucl. Inst. Meth. A 528 (1999) p.570.

[21] E.M. Lauridsen, D. Juul Jensen, H.F. Poulsen, U. Lienert, Scripta Mater. 43 (2000) p.561.

[22] E.M. Lauridsen, S. Schmidt, S.F. Nielsen, L. Margulies, H.F. Poulsen, D. Juul Jensen, Scripta Mater. 55 (2006) p.51.

[23] S.O. Pouslen, E.M. Laudrisen, A. Lyckegaard, J. Oddershede, C. Gundlach, C. Curfs, D. Juul Jensen, Scripta Mater. 64 (2011), p.1003.

[24] R.B. Godiksen, S. Schmidt, D. Juul Jensen, Scripta Mater. 57 (2007) p.345.

[25] R.A. Vandermeer, N. Hansen, Acta Mater. 56 (2008) p.5719.

[26] H.W. Zhang, X. Huang, R. Pipan, N. Hansen, Acta mater. 58 (2010) p.1698.

[27] R.A. Vandermeer, D. Juul Jensen, Acta Mater. 49 (2001) p.2083.

[28] G. Wu, D. Juul Jensen, Mater. Sci. Tech. 21 (2005) p.1407. 


\section{Figure Captions:}

Figure 1 EBSD orientation maps of the microstructure a), after deformed to a true strain of 4, b), after $5 \mathrm{~h}$ annealing at $257^{\circ} \mathrm{C}$, and c), after $20 \mathrm{~h}$ in-situ annealing at $257^{\circ} \mathrm{C}$. In the maps, colours represent crystallographic orientations expressed by three Euler angles with colour legends shown in d). Black lines represent boundary misorientations $\geq 15^{\circ}$, and light grey lines $\geq 1^{\circ}$ and $<15^{\circ}$.

Figure 2: Schematic diagram of the in-situ 3DXRD experiment setup.

Figure 3: (a) Recrystallisation kinetic curves of 10 individual crystallites during annealing at $257{ }^{\circ} \mathrm{C}$ and, (b), size distribution of all the 170 crystallites after annealing $257^{\circ} \mathrm{C}$ for $20 \mathrm{~h}$. The average size is $9.8 \mu \mathrm{m}$.

Figure 4: Distribution of sizes of all crystallites after annealing for $0.7 \mathrm{~h}$. The average size is $3.2 \mu \mathrm{m}$. The blue and red coloured ones are the ones end up having large and small sizes, respectively (i.e. above or below the average of $9.8 \mu \mathrm{m}$ ).

Figure 5: Distributions of growth rates as a function of size of all crystallites after, (a), $0.7 \mathrm{~h}$ and, (b), $5.0 \mathrm{~h}$ annealing; (c) Average growth rate of recrystallising and recovering crystallites during annealing.

Figure A1: (a) A diffraction image obtained by the CCD detector during annealing. (b) Intensity spectrum for 6 overlapping peaks (black line) and each peak after the multiple Gaussian curve fitting (coloured lines). 
Dear editor,

Thank you very much for your very careful reviewing our manuscript submitted to Phil. Mag. entitled "In-situ measurement of annealing kinetics of individual bulk grains in nanostructured aluminium”.

According to the suggestions of the two referees, we have revised our manuscript following all the suggestions. The revisions are listed below. The revisions are also marked in red in the text of the manuscript.

\section{Referee 1:}

1. Q: For a detailed description of the method it is referred to a paper [\#6] to be published.

A: The paper referred to be published, old Ref [6], is replaced by published results on the same material, new Ref. [6].

2. Q: The first paragraph of the introduction appears to be rather weak.

A: This paragraph has been rewritten and we now put more emphasize on the potential of the technique to characterize nanostructured alloys.

3. Q: There is always a space between the number and the unit.

A: A space is added between a number and its unit.

4. Q: Please provide more detail on the processing parameters of the material.

A: The material was provided by Alcan, and was rolled at Alcan by industrial standards. As for the detailed processing parameters, we are sorry to tell that we don't know exactly how the material was rolled. However, we mentioned in the text that this material was provided by Alcan, and chemical data are given.

5. Q: The sequence of numbering of the figures.

A: We correct the sequence of all figures in the on-line submission system. I hope this time everything works well.

6. Q: The materials composition should be given in detail.

A: The chemical composition of the material is included in the text.

7. The discussion should also address a rough estimate about the driving forces for grain coarsening and recrystallization and how it is related to the grain boundary mobility.

A: Our present experimental results show that the growth rates of individual grains vary. In other work, it is shown that the activation energies of individual grains also vary (Ref [23]). Therefore boundary mobilities calculated from activation energies also vary from grain to grain. We can't say if the variation of growth rates observed in the present paper relates to variations of mobility or local driving pressure (stored energies). To calculate the mobility of each crystallite, the stored energy ahead of each crystallite is needed, which is not possible to be determined by the present technique. In other words, it is not possible to estimate the boundary mobility or activation energy from the present 3DXRD data.

8. Q: The following figs need to be revised.

A: Figure 3a is re-plotted by choosing only 10 examples to clearly show the recrystallising / coarsening kinetics of individual crystallites. Thus Figure 3 c is deleted. As for figure $3 b$ and 4 , we 
intended to show the differences between "Large" grains and "Small" grains. We think the frequency plot can show the differences more clearly than the accumulative frequency curves. These figures, Fig $3 \mathrm{~b}$ and 4, are not changed. The contrast of Figure $1 \mathrm{~A}$ is modified. But as the diffraction spots of crystallites determined by synchrotron are always very sharp and tiny, they will never appear very clearly.

\section{Referee 2:}

1. Q: The color projection of the crystal orientations is missing.

A: The colour legend of the Euler orientation map is added in Fig 1.

2. Q: Are images in Fig. 1 taken from reference?

A: The figures in this manuscript were obtained from the exact same material to that in the paper to be published elsewhere. But the figures are different.

3. Q: Is "s" in the phrase "...Al cold rolled to s strain of 4.0 coarsening by recovery...", page 4 line 11 a mistype or refers to a value?

A: ' $s$ ' is a typo, and it was revised to ' $a$ '.

4. Q: The abbreviation RD is used (p. 5 line 1) before it is defined later on the same page.

A: This is revised and $\mathrm{RD}$ is defined when it is used the first time.

5. Q: Page 12, line 3 - probably the word "continues" instead of "continuous" should be used.

A: The word 'continuous' was replaced by 'continues'.

6. Q: Error bars or equivalent quantity related to the obtained results should be stated in order for the reader to be able to assess the confidence interval, e.g. Fig. 5c.

A: Error bars are added in the new figure 5c.

7. Q: Fig. 2 is rather small and unclear; its omission may be considered, especially as it is the same figure as in ref. [13].

A: Figure 2 can help reader to follow the experimental procedures more easily. We prefer to keep the figure, but we made it bigger so that reader can read it easily.

8. Q: The sequence of figures in the manuscript pdf is not ordered; fig. captions also show no figure number, which may lead to confusion.

A: The sequences of figures and figure number were revised in the on-line submission system.

9. Q: What does the legend in Fig. 5 (ReX, ReV) stand for?

A: ReX and $\operatorname{ReV}$ are replaced by Recrystallisation and Recovery.

10. Q: Information related to the synchrotron facility used and further relevant details are also desirable, e.g. as per ref. [13].

A: It is true that relevant details on the synchrotron facility are very useful, especially for readers who are not familiar to the technique. However, Ref [13] and other articles already explained the technique in great details. In the present article, we give essential information of the experiments. Other details of the technique, which are not directly related to the experimental results, are not given.

11. Q: Section Experimental mentions 300 1s exposures for the given omega interval, omega step and oscillation. How is this related to the statement that temporal resolution of 0.7 hour is obtained?

A: It is correct that the time to finish 300 1s exposures is less than 0.7 hours. In our experiments, we did two scans for each step. One scan is used for the present annealing kinetics experiment; the other is not related to the present paper. This is clarified in the text. 
12. Q: Can the results of velocity-time dependences be used for assessment of the stated values of activation energy, even in spite of the fact that only a single annealing temperature was reported?

A: As stated above in Q7, determination of boundary mobility and activation energy is not possible from the present experiments.

13. Q: A more detailed/accurate description of Fig. 4 would be desirable, especially describing the "first" and the "second" scan - what is meant by this in the manuscript text?

A: The figure 4 is re-plotted and the description of the figure is also revised in the text.

We hope that our revisions are satisfactory so our manuscript can be accepted for the publication on Phil. Mag., and are looking forwards to hearing from you.

Yours sincerely,

Guilin Wu and Dorte Juul Jensen 